\title{
New Flexible Payment Plans for member and non-member dentists
}

Simplyhealth Professionals has launched a new flexible payment plan for both its member practices and non-members which will help patients to spread the cost of both dental and facial aesthetic treatments. They will also offer practices the ability to generate new and regular income streams.

The new Flexible Payment Plans will make treatments more accessible and affordable for all patients as they can set the price and payment length with their dentist so it suits individual budgets. Patients can opt for treatments that they might have previously thought were unaffordable.

Dentists will agree with each patient how much they pay each month and how long their treatment will take. They can offer the patient an ongoing monthly plan for more regular treatments, or a choice of three to ten monthly payments for a one-off treatment, helping the patient to spread the cost of treatment and make it more affordable for them.

This is the first time that Simplyhealth Professionals has created a payment plan that can be tailored to support dentists providing an increasingly diverse mix of cosmetic dental and facial aesthetic treatments in their practices. Flexible Payment Plans will encourage patients to opt for new or higher cost treatments which they previously might not have considered due to the price.

\section{Greater flexibility}

However, dentists do not have to be a Simplyhealth Professionals member dentist to offer Flexible Payment Plans. For the first time, non-members will be able to take advantage of this payment plan from the company. This will appeal to those dentists who specialise in high end treatments such as facial or cosmetic work who want a flexible plan to cover this treatment, in addition to traditional restorative and preventive dental treatments.

Sandy Brown, Director of Dentists at Simplyhealth Professionals, said: 'We wanted to be able to offer all dentists greater flexibility and choice for their patients beyond traditional routine dental treatments. Our new Flexible Payment Plans give dentists the freedom to provide exactly what their patients ask for and help their patients to spread the cost of treatment. As the plans can be used for non-dental treatments such as facial aesthetics, it opens up a wider patient market for practices.'

What will you use your plan for?

- Teeth straightening

- Anti-wrinkle treatment

- Dermal fillers

- Implant maintenance

- Whitening

- Facial aesthetics

It really is up to you

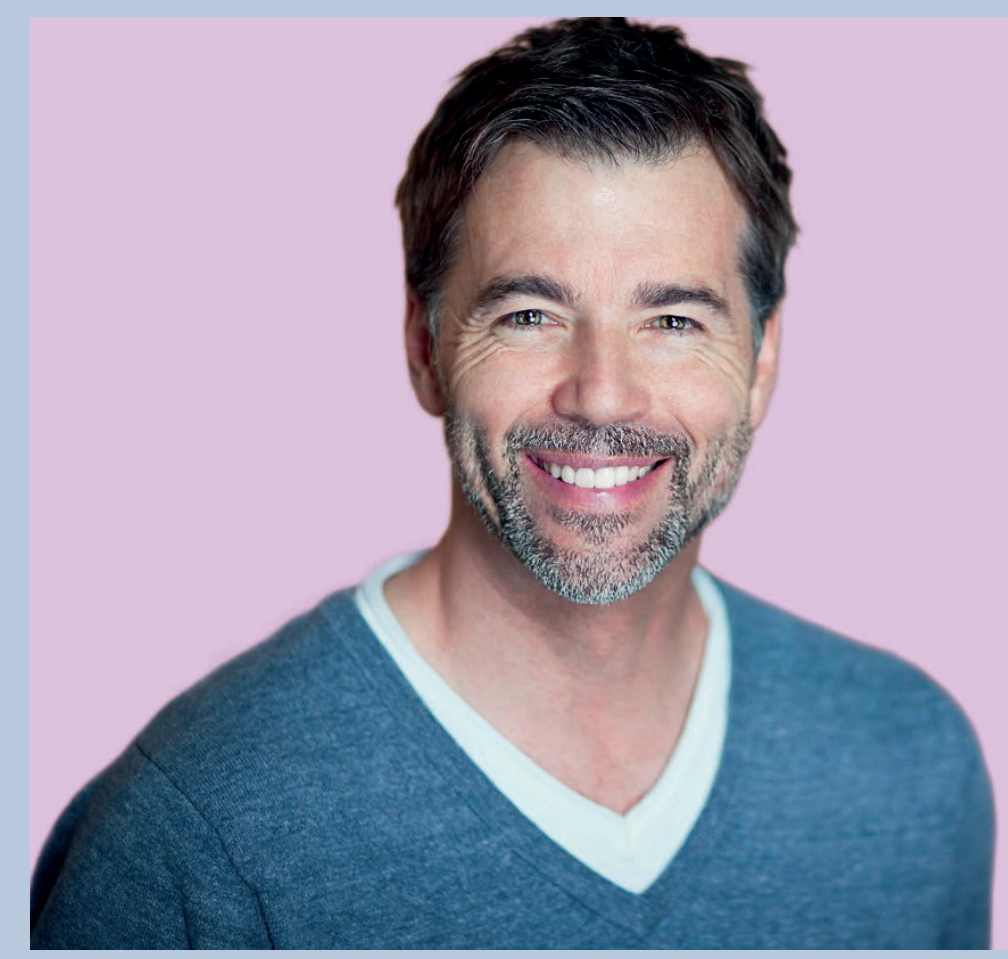

\section{Greater choice}

Flexible Payment Plans are particularly well suited for more costly treatments and non-routine treatments such as tooth whitening and straightening, implant maintenance, facial aesthetics and cosmetic dentistry, or as a restorative treatment plan for crowns, bridges and dentures. It will open up greater choice for patients in non-dental treatments such as anti-wrinkle or dermal fillers. Dentists can also create, brand and promote their own Hygiene or Whitening Plans using the Flexible Payment Plans platform.

The plans are incredibly straightforward and can be created immediately in the practice. Once the dentist has agreed the treatment plan and costs with their patient, they set up the plan on the Flexible Payment Plan portal. Once the patient's personal and payment details are entered, the plan is immediately set up and ready to start, and the patient can book appointments for their treatment. The dentist can access the portal at any time to check the status of an individual plan.

Practices can also use Simplyhealth Professionals' online design service to create bespoke posters and literature for individual flexible payment plans. There are currently six different posters for them to choose from.

For more information on Flexible Payment Plans call 03306780155 or visit www.denplan.co.uk

The content of this promotion is the responsibility of the advertiser, Simplyhealth Professionals. 